\title{
ANÁLISE DA CAPACIDADE FUNCIONAL E DOR EM PACIENTES QUE REALIZAM HEMODIÁLISE
}

Paula de Barros; Isabela Malaguti; Thamara Müller Santos; Daiane Carolina Nascimento dos Santos; Layane Lopes Napoleão; Renata Calciolari Rossi e Silva, Susimary Aparecida Trevizan Padulla

Faculdade de Ciências e Tecnologia-FCT/Unesp, Presidente Prudente, SP, Brasil- Pró Reitoria de Extensão Universitária-PROEX. E-mail: paulamanfrim@gmail.com

\section{RESUMO}

A Doença Renal Crônica (DRC) leva a incapacidade renal em manter a homeostasia interna do organismo. Após o início do tratamento dialítico o cotidiano deste indivíduo se torna monótono, restrito, favorece o sedentarismo comprometendo suas atividades de vida diária (AVD's). Analisar a capacidade funcional e intensidade da dor nos pacientes que realizam hemodiálise. Participaram 115 pacientes avaliados através dos questionários: Índice de Barthel (IB) utilizado para mensurar o grau de dependência funcional nas AVD's e a Escala Visual Analógica (EVA) para quantificar a intensidade de dor. Comparando as variáveis do IB, foi observada diferença significativa entre os grupos dependência leve, total e severa. Quanto à EVA, foi observada diferença significativa entre dor intensa comparada com dor leve e moderada com dor intensa e leve. Os resultados encontrados neste estudo foram positivos quanto à capacidade funcional e intensidade leve de dor nos pacientes que realizam hemodiálise.

Palavras-chave: Doença renal crônica, hemodiálise,capacidade funcional,dor

\section{INTRODUÇÃO}

A fisiopatologia da Doença Renal Crônica (DRC) é a perda progressiva da função renal devido à deterioração e destruição dos néfrons, que são as unidades funcionais dos rins ${ }^{(1)}$ Quando os rins perdem sua capacidade de realizar sua função, eliminar as toxinas que são liberadas pelo metabolismo, é necessário submeter o doente a um tipo de tratamento que substitui a função renal $^{(2)}$.

Dentre as principais doenças que alteram a função renal estão a glomerulonefrite crônica, anemias, hipertensão arterial, processos renais obstrutivos crônicos, diabetes mellitus e doenças hereditárias ${ }^{(3)}$.

Em resumo a DRC é o resultado final de múltiplos sinais e sintomas decorrentes da incapacidade renal de manter a homeostasia interna do organismo, e, uma vez instalada, é necessário um tratamento contínuo para substituir a função renal ${ }^{(4)}$. A DRC é considerada uma condição sem alternativas de melhoras rápidas, de evolução progressiva, causando problemas médicos, sociais e econômicos, já que também atinge jovens em idade produtiva ${ }^{(5)}$. 
No Brasil, aproximadamente 54,5 mil pessoas encontram-se em terapia dialítica, das quais cerca de 49 mil em hemodiálise e 5,5 mil em diálise peritoneal. Esse número cresce, em média, $10 \%$ ao ano, por conta de uma incidência de mais de cem novos pacientes por milhão de habitantes ${ }^{(6)}$.

O tratamento da DRC envolve métodos de substituição renal incluindo o transplante renal, a diálise peritoneal e a hemodiálise.

O transplante renal é a substituição dos rins doentes por um rim saudável de um doador. É o método mais efetivo e de menor custo para a reabilitação de um paciente com insuficiência renal crônica terminal ${ }^{(7)}$.

O tratamento mais utilizado denomina-se hemodiálise. Este tratamento é definido como um procedimento que limpa e filtra o sangue, liberta o corpo dos resíduos prejudiciais, do excesso de sal e de líquidos, controlando a pressão arterial e ajudando o organismo a manter o equilíbrio de substâncias químicas como o sódio, o potássio e cloretos ${ }^{(8)}$.

O portador de DRC convive com uma doença que o obriga a submeter-se a um tratamento doloroso, de longa duração que provoca muitas limitações e complicações advindas da terapia dialítica e da própria enfermidade renal. Dentre elas estão a diminuição da atividade física ${ }^{(5,9,10)}$.

Após o inicio do tratamento hemodialítico o cotidiano do indivíduo se torna monótono e restrito, limitando suas atividades favorecendo o sedentarismo ${ }^{(11)} \mathrm{Com}$ a diminuição da atividade física a capacidade funcional também é diminuída e tem como alterações físicas a atrofia muscular, fraqueza, cansaço e edema de membros, dor lombar e outros, dificultando a realização das atividades de vida diária (AVD's) ${ }^{(12)}$.

A doença renal causa elevadas taxas de morbimortalidade e tem significativo impacto negativo sobre a qualidade de vida relacionada à saúde ${ }^{(5)}$ tais transtornos na vida cotidiana do paciente têm motivado estudos sobre a qualidade de vida. Mas pouco se sabe a respeito da reabilitação deste paciente durante a hemodiálise ${ }^{(11)}$.

\section{OBJETIVO GERAL}

- Analisar a capacidade funcional e intensidade da dor nos pacientes que realizam hemodiálise.

\section{OBJETIVOS ESPECÍFICOS}

- Mensurar a dependência funcional dos pacientes com doença renal crônica através do Índice de Barthel 
- Quantificar a intensidade de dor através da Escala Visual Analógica

\section{METODOLOGIA}

Foram incluídos na pesquisa 115 pacientes, de ambos os sexos, conscientes, orientados. E foram excluídos, todos os pacientes portadores de doenças neurológicas ou complicações, que pudesse inviabilizar a aplicação dos questionários.

Os participantes responderam a dois questionários que foram aplicados em forma de entrevista individual durante as sessões de hemodiálise. Para tal foram utilizados os questionários Índice de Barthel com o objetivo de mensurar a dependência funcional e a Escala Visual Analógica (EVA) foi utilizada para quantificar a dor.

Este projeto de pesquisa foi avaliado e aprovado pelo Comitê de Ética em Pesquisa Humana da Universidade Estadual Paulista Julio de Mesquita Filho, campus Presidente Prudente (UNESP) com o protocolo de n. 97/2011. Também recebeu da Instituição do Rim da Santa Casa de Misericórdia de Presidente Prudente - SP, o consentimento formal para a realização da pesquisa.

\subsection{Avaliação da Dependência Funcional}

O questionário Índice de Barthel foi utilizado para avaliar o grau de dependência funcional. Cada item é pontuado de acordo com o desempenho do paciente em realizar tarefas, de forma dependente, com alguma ajuda ou de forma independente. Uma pontuação geral é formada atribuindo-se pontos em cada categoria, a depender do tempo e da assistência necessária a cada paciente. A pontuação varia de 0 a 100, em intervalos de cinco pontos, e as pontuações mais elevadas indicam maior independência nas AVDs avaliadas. No contexto clínico o IB nos dá informações importantes, não só a partir da pontuação total, mas também a partir das pontuações parciais para cada atividade avaliada, porque permitem conhecer quais são as incapacidades específicas da pessoa e como tal adequar os cuidados às necessidades ${ }^{(13)}$.

\subsection{Avaliação da Dor}

A Escala Visual Analógica (EVA) consiste no auxílio da aferição da intensidade da dor no paciente. Deve-se questionar ao indivíduo quanto ao seu grau de dor sendo que os valores variam de $0^{(14)}$. correspondente à ausência de dor, e 10 como sendo o nível máximo da intensidade da dor. Foi estabelecido como método para a aplicação desta escala o 
seguinte questionamento: "Por favor, me diga qual é a intensidade de dor que o senhor (a) está sentindo neste exato momento, lembrando que a escala varia de 0-10 sendo que 0 corresponde a nenhuma dor e 10 a uma dor extremamente forte".

Para analisar os resultados foi confeccionado um banco de dados eletrônico e para a análise foi utilizado o programa sigma stat 2.0. Foram considerados significativos os valores em que $\mathrm{p}$ for menor que 0,05 .

\section{RESULTADOS}

Um número de 115 pacientes foi avaliado e a média de idade atingida foi de 57,6 anos.

$\mathrm{Na}$ comparação entre as variáveis do índice de Barthel, foi observada diferença significativa entre os grupos dependência leve e total; dependência leve com dependência severa e dependência leve com moderada apresentando valores de $p$ menores que 5\%. (Tabela 1). Em relação à escala analógica de dor, foi observada diferença significativa entre os pacientes que sentiram dor intensa comparada com dor leve e na variável dor moderada comparada com dor intensa e leve (Tabela 2).

Tabela 1. Comparação entre as variáveis do índice de Barthel em 115 indivíduos que realizam hemodiálise na Santa Casa de Misericórdia da cidade de Presidente Prudente - SP.

\begin{tabular}{lll}
\hline Grupos & $\mathrm{n}(\%)$ & Med (máx- min) \\
\hline Dependência Leve & $84,0(73,0)$ & $85,0(85,0-85,0)$ \\
Dependência Moderada & $24,0(20,9)$ & $72,0(67,5-73,5)^{*}$ \\
Depedência Severa & $4,0(3,5)$ & $39,5(32,0-45,5)^{*}$ \\
Dependência Total & $3,0(2,6)$ & $8,0(2,0-17,0)^{*}$ \\
Totalmente Independente & $0,0(0,0)$ & \\
Total & $115(100)$ \\
\hline $\mathrm{H}=82,863 ; p=<0,001$. Dunn's Method: $\mathrm{p}<0,05^{*}$ : D Leve vs D Total; D Leve vs D Severa; D Leve vs D Moderada
\end{tabular}

Tabela 2. Comparação entre as variáveis da escala analógica de dor em 115 indivíduos que realizam hemodiálise na Santa Casa de Misericórdia da cidade de Presidente Prudente - SP

\begin{tabular}{lll}
\hline Grupos & $\mathrm{n}(\%)$ & Med (máx- min) \\
\hline Dor moderada & $39(33,9)$ & $5,0(4,0-5,0)^{*}$ \\
Dor leve & $59(51,3)$ & $0,0(0,0-0,0)^{*}$ \\
Dor intensa & $17(14,8)$ & $10,0(10,0-10,0)^{*}$ \\
Total & $115(100)$ & \\
\hline
\end{tabular}

$H=106,665 ; p=<0,001$. Dunn's Method: $p<0,05^{*}$ : intensa vs leve, intensa vs moderada, moderada vs leve

\section{DISCUSSÃO}


De acordo com estudo realizado por Kusumota et al ${ }^{(4)}$., há maior incidência na população adulta do sexo masculino, demonstrando tendência geral de aumento contínuo e progressivo de idosos com doença renal crônica, visto que a idade avançada é considerada um dos fatores que justificam o aumento de pacientes em hemodiálise.

Os achados deste estudo quanto à dependência funcional avaliada através do índice de Barthel nos mostram que [n(\%) 84,0 $(73,0)]$, apresenta uma dependência leve para a realização de suas atividades de vida diária. Sendo que este número cai para [n(\%) 24,0 (20,9)] com dependência moderada, o que nos leva a crer que a diminuição da atividade física que tem como consequência alterações física está mais para o cotidiano do indivíduo que se torna monótono e restrito do que para seu grau de dependência funcional ${ }^{(11)}$.

Em concordância com o estudo de Oller et al.,2012 ${ }^{(15)}$ apesar de os resultados apontarem para uma dependência leve para a maior parte dos pacientes avaliados, é relevante considerar que os pacientes apresentaram algum grau de dependência, para locomoção ao subir e descer escadas e vestirem-se especialmente pelas características da população estudada.

Este mesmo autor afirma que a manutenção da independência funcional pode ter implicações importantes para melhorar a qualidade de vida, e essa independência funcional também está relacionada com a capacidade dos pacientes em realizar atividades prazerosas e atividades que são essenciais dentro de sua rotina diária.

Corroborando com nosso estudo estes mesmos autores nos dizem que a própria DRC e seu tratamento podem causar limitações físicas e emocionais, o que interfere na vida dos pacientes, limitando ou mesmo impedindo a realização de tarefas diárias ${ }^{(15)}$.

A Escala Visual Analógica foi utilizada para quantificar a intensidade de dor naquele momento em que os indivíduos realizavam a hemodiálise. De acordo com os resultados obtidos neste estudo vimos que entre [n (\%)115 (100)] pacientes [n (\%)59 (51,3)] apresenta dor considerada leve e um número de apenas [n (\%)17 (14,8)] apresenta dor considerada intensa de acordo com a Escala Visual Analógica.

Com esse resultado podemos analisar a possibilidade de uma intervenção fisioterapêutica realizada durante a terapia dialítica, já que na literatura podemos encontrar estudos feitos com essa população e intervenções nas diversas áreas da fisioterapia.

De acordo com Soares et al ${ }^{(11)}$ a realização de exercícios durante a diálise aumenta a tolerância ao exercício e a qualidade de vida dos pacientes, além de contribuir para o retorno às atividades de vida diária e dar motivação em um ambiente estruturado e monótono. 


\section{CONCLUSÃO}

Os resultados encontrados neste estudo foram positivos quanto à capacidade funcional e intensidade leve de dor nos pacientes que realizam hemodiálise.

Portanto, ressalta-se a importância da atuação fisioterapêutica com o intuito de trazer mais benefícios para a população estudada e amenizar este impacto negativo que a doença renal crônica e o tratamento dialítico causam nestes indivíduos.

Em conclusão vemos a necessidade de mais estudos para avaliar as capacidade funcional e limitações desta população assim como a qualidade de vida.

\section{REFERÊNCIAS}

1. Guyton A, Hall J. Tratado de fisiologia Médica. Rio de Janeiro:Guanabara Koogan, 2006.

2. Andolfato C, Mariotti MC. Avaliação do paciente em hemodiálise por meio da medida canadense de desempenho ocupacional. Vol 20, n1.p. 1-7, jan/abr. 2009.

3. Macedo LO, Batista GM, Brasileiro ME. Indicadores de qualidade de vida em pacientes renais crônicos submetidos à hemodiálise. Rev Eletr Enferm 2010. Disponível a partir de: http://www.ceen.com.br/revista eletronica.

4. Kusomota L, Rodrigues RAP, Marques S. Idosos com insuficiência renal crônica: alterações do estado de saúde. Rev. Latino-Am. Enferm, Ribeirão Preto 2004;12(3).

5. Martins MRI, Cesarino CB. Qualidade de vida de pessoas com doença renal crônica em tratamento hemodialiticos. Rev. Latino-Am. Enferm, 13(5):670-676, set/out, 2005.

6. Vieira WP, Gomes KWP, Frota NB, Andrade JECB, Vieira RMRA, Moura FEA, Vieira, FJF. Manifestações musculoesqueléticas em pacientes submetidos à hemodiálise. Rev Bras Reumatol. 2005; 45(6):357. http://dx.doi.org/10.1590/S0482-50042005000600005

7. Busato O. Transplante Renal, 2001. Disponível em < http://www.abcdasaude.com.br/artigo.php?418> Acesso em: 17 de novembro de 2011.

8. Agripino AS, Sá AKG.Perfil de pacientes que realizam hemodiálise no Sertão Central Pernambucano.Disponível em:

http://www.fachusc.com.br/artigos tecnicos/Perfil de pacientes que realizam hemodialise no sertao central PE.pdf> Acesso em: 24/08/2013.

9. Gullo ABM, Lima AFC, SIlva MJP. Reflexões sobre comunicação na assistência de enfermagem ao paciente renal crônico. Ver.Esc.Enferm.USP São Paulo 2000; 34(2): 209-212.

10. Padulla SAT, Matta MV, Melatto T, Miranda RCV, Camargo MR.A fisioterapia pode influenciar na qualidade de vida de indivíduos em hemodiálise? Ver Ciên, cuid e saú 2011; 10(3): 564-570. 
11. Soares A, Zehetmeyer M, Rabuske M. Atuação da Fisioterapia durante a Hemodiálise Visando a Qualidade de Vida do Paciente Renal Crônico. Rev. de Saú UCPEL, Pelotas.1(1)Jan/Jun. 2007.

12. Yen LS, Padulla SAT, Miranda RCV, Fahur BS, Sato KT. Avaliação da capacidade funcional e escala de dor associadas ao exercício físico em pacientes com doença renal crônica que realizam hemodiálise. Presidente Prudente- SP, 2010.

13.Minosso JSM, Amendola F, Alarenga MRM, Oliveira MAC. Validação, no Brasil, do Índice de Barthel em idosos atendidos em ambulatórios.Acta Paul Enferm,São Paulo;23(2):218-23. 2010. http://dx.doi.org/10.1590/S0103-21002010000200011

14.Yen LS, Padulla SAT, Miranda RCV, Fahur BS, Sato KT, Ferrari GNB. Efeito do exercício físico sobre a capacidade funcional e escala de Borg em pacientes com doença renal crônica,Presidente Prudente,2010.

15. Oller GASAO, Ribeiro RCHM, Travagi DSA, Batista MA, Marques S, Kusumota L. Independência funcional em pacientes com doença renal crônica em tratamento hemodialítico. Rev. Latino-Am. Enfermagem 20 (6) :[08] nov.-dez. 2012 